\title{
Gibbons-Hawking Boundary Terms and Junction Conditions for Higher-Order Brane Gravity Models
}

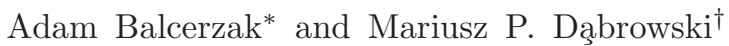 \\ Institute of Physics, University of Szczecin, Wielkopolska 15, 70-451 Szczecin, Poland.
}

(Dated: October 24, 2018)

\begin{abstract}
We derive the most general junction conditions for the fourth-order brane gravity constructed of arbitrary functions of curvature invariants. We reduce these fourth-order theories to second order theories at the expense of introducing new scalar and tensor fields - the scalaron and the tensoron. In order to obtain junction conditions we apply the method of generalized Gibbons-Hawking boundary terms which are appended to the appropriate actions. After assuming the continuity of the scalaron and the tensoron on the brane, we recover junction conditions for such general brane universe models previously obtained by different methods. The derived junction conditions can serve studying the cosmological implications of the higher-order brane gravity models.
\end{abstract}

PACS numbers: 98.80.Cq, 04.50.-h, 11.25.Mj

\section{INTRODUCTION}

In the simplest approach to the variational principle of any theory of gravity, such as Einstein relativity, one used to assume that both the variation of the metric tensor $\delta g_{a b}$, and the variation of the first derivative of the variation of the metric tensor $\delta g_{a b ; c}$, vanish on the boundary of the integration volume [1]. However, motivated by quantum cosmology, Gibbons and Hawking 2 claimed that the latter requirement is too strong, and so they suggested exact cancellation of the term coming from the variation of the action which involves the derivatives with a postulated extra boundary term - nowadays called just the Gibbons-Hawking boundary term. For Einstein relativity the Gibbons-Hawking boundary term is constructed of the trace of the extrinsic curvature [2]. It is expected that any theory of gravity may be appended by an appropriate boundary term. Hawking and Lutrell $[3$ found a boundary term for the fourth order gravity theory composed of the combination of the square of the Weyl tensor and an arbitrary function of the scalar curvature in four dimensions and studied its Wheeler-deWitt quantization. The boundary terms for gravity theory of an arbitrary function of the scalar curvature were also studied by Barrow and Madsen [4] and in a more general case of an arbitrary function of curvature invariants by Barvinsky and Solodukhin [5]. The study of the appropriate boundary terms for the Gauss-Bonnet density being one of the general Lovelock densities [6, 7] has also been done [8, 9, 10, 11]. These densities are, however, special combinations of the curvature invariants which give the second-order field equations. The Gibbons-Hawking boundary term for Lovelock gravity with AdS asymptotics has been found, too [12].

Brane gravity theories initiated by Hořava and Witten [13] and further developed by Randall and Sundrum [14]

\footnotetext{
*Electronic address: abalcerz@wmf.univ.szczecin.pl
}

†Electronic address: mpdabfz@wmf.univ.szczecin.pl add an extra aspect to the problem of boundary terms, since the boundary of the bulk spacetime is the brane, which serves as the actual universe [15]. In analogy to a surface layer problem of electromagnetism, in brane models, one has to study appropriate junction conditions [16]. The problem of the gravitating surface layer junction conditions was first solved by Israel [17], and recently applied to brane universes in Gauss-Codazzi formalism 18]. It was also solved for a Brans-Dicke braneworld [19]. All these considerations became the basic conditions for studying cosmological solutions within the framework of brane scenario which allows the modification of the Newton's law on small scales. Brane models produce a different cosmological framework, since the square in energy density term and the dark radiation resulting from transferring the gravitons from the bulk to the brane and vice versa appear in generalized cosmological equations [18].

Within the physically interesting context, it seems challenging to formulate braneworld scenario for generalized gravity theories such as Lovelock gravity and the fourth-order gravity. In order to achieve that, one necessarily has to formulate Israel junction conditions for these gravities on the brane. In fact, for the Gauss-Bonnet brane, these conditions were formulated by the application of the Gauss-Codazzi formalism in Ref. 20]. This allowed many detailed studies of the Gauss-Bonnet brane cosmologies 21, 22, 23, 24], and of general Lovelock cosmologies 25]. However, Lovelock brane models are free from the problem of divergencies resulted from the appearance of the powers of the delta function in the field equations 25, 26]. In fact, gravity theories which are based on the lagrangians being the functions of curvature invariants such as $f(R)$ theory (see 27]; for a recent review see [28] ) or $f\left(R, R_{a b} R^{a b}, R_{a b c d} R^{a b c d}\right)$ theory [29, 30], unavoidably lead to such divergencies and the formulation of the junction conditions is a non-trivial task. In Ref. 26 we have proposed the resolution of the problem for such theories by imposing more regularity onto the metric tensor at the brane position, though still keeping the theory to be a fourth-order. However, such a strong regularity of the metric at the brane may 
seem somewhat restrictive and so we have also explored the equivalence of these conditions to the conditions obtained for an equivalent second-order theory with an extra scalar degree of freedom - the scalaron [31]. We have also assumed the continuity of the scalaron on the brane. Such a correspondence for surface layer $f(R)$ universes has already been studied in Refs. [32, 33] and quite recently in Ref. [34] in the Gauss-Codazzi approach. On the other hand, in Ref. [35] the gravity theory of the linear combination $f\left(R, R_{a b}, R_{a b c d}\right)=$ $a R^{2}+b R_{a b} R^{a b}+c R_{a b c d} R^{a b c d}$ ( $a, b, c=$ const.) was studied in the Gibbons-Hawking boundary term approach. In these references some example cosmological solutions have been found. Our current task is to extend these considerations onto $f\left(R, R_{a b} R^{a b}, R_{a b c d} R^{a b c d}\right)$ theory by using the Gibbons-Hawking boundary term method, also in the most general case, where the discontinuity of the new fields - the scalaron and the tensoron - at the brane position is allowed.

In Section [II we discuss junction conditions for the fourth-order $f(R)$ gravity theory (after transforming it to the second order theory) by adding an appropriate Gibbons-Hawking boundary term, constructed of the extrinsic curvature and an extra scalar field - the scalaron. In Section [II we derive junction conditions for a general $f\left(R, R_{a b} R^{a b}, R_{a b c d} R^{a b c d}\right)$ gravity theory, first by transforming it to the second-order theory, and then by adding an appropriate Gibbons-Hawking boundary term, which is constructed of the extrinsic curvature and an extra rank four tensor field - the tensoron. In fact, we benefit from the discussion of general junction conditions for these higher-order theories following our complementary approach given in Ref. [26], but we derive more general junction conditions - the ones which do not possess continuity of the scalaron and the tensoron at the brane. In Section IV we give our conclusions.

\section{GIBBONS-HAWKING BOUNDARY TERM AND JUNCTION CONDITIONS FOR F(R) GRAVITY}

As the first example of the application of the GibbonsHawking boundary term method to derive junction conditions for brane universes, we discuss the $f(R)$ gravity theory in $D$ spacetime dimensions

$$
S_{p}=\chi^{-1} \int_{M_{p}} d^{D} x \sqrt{-g} f(R)+S_{b u l k, p}
$$

where $R$ is the Ricci scalar, $\chi$ is a constant, $S_{b u l k . p}$ is the bulk matter action, and $p=1,2$. It is known [33] that this theory gives fourth-order field equations and that it can be expressed in an equivalent way by using the following action

$$
\begin{aligned}
\bar{S}_{p} & =\int_{M_{p}} d^{D} x \sqrt{-g}\left\{f^{\prime}(Q)(R-Q)+f(Q)\right\} \\
& +S_{b u l k, p}
\end{aligned}
$$

where $M_{p}$ is the spacetime volume, $Q$ is an extra field (a lagrange multiplier), $K$ is the trace of the extrinsic curvature, $h$ is the determinant of the induced metric and $f^{\prime}(Q)=d f(Q) / d Q$. Varying (II.2) with respect to $Q$, one obtains an equation of motion $Q=R$ (provided $f^{\prime \prime}(Q) \neq$ 0 ), and this is equivalent to a scalar-tensor Brans-Dicke gravity with Brans-Dicke parameter $\omega_{B D}=0$, and the potential $V(H)=-H Q(H)+f(Q(H))$ (see e.g. 31]). Defining $H=f^{\prime}(Q)$ (a scalaron [31]) one can rewrite (II.2) in the form

$$
\bar{S}_{p}=\int_{M_{p}} d^{D} x \sqrt{-g}\{H R-V(H)\}+S_{b u l k, p} .
$$

The variation of (II.3) gives

$$
\begin{aligned}
\delta \bar{S}_{p} & =\int_{M_{p}} d^{D} x \sqrt{-g}\left\{\left[\frac{1}{2} g^{a b} H R-H R^{a b}\right.\right. \\
& -\frac{1}{2} g^{a b} V(H)+H_{; d c}\left(g^{a b} g^{c d}-g^{(a c} g^{b) d}\right) \\
& \left.\left.-\frac{\chi}{2} T_{b u l k, p}^{a b}\right] \delta g_{a b}+\left(R+\frac{\partial V}{\partial H}\right) \delta H\right\} \\
& +\int_{\partial M_{p}} d^{D-1} x \sqrt{-h} 2 H \underline{n_{d} ; c} g^{a[b} g^{c] d} \delta g_{a b} \\
& +\int_{\partial M_{p}} d^{D-1} x \sqrt{-h} 4 H_{;(c} \underline{n_{d)}} g^{a[b} g^{c] d} \delta g_{a b} \\
& -\int_{\partial M_{p}} d^{D-1} x \sqrt{-h}\left(2 H g^{a[b} g^{c]} \underline{n_{d}} \delta g_{a b}\right)_{; c},
\end{aligned}
$$

where $\underline{n^{a}}$ is a unit vector normal to $\partial M$, and it is chosen to be "outward pointing", if spacelike, and "inward pointing", if timelike (this is according to the Gauss integral theorem), and the induced metric is given by $h_{a b}=g_{a b}-\epsilon n_{a} n_{b}(\epsilon=1$ for a timelike brane, and $\epsilon=-1$ for a spacelike brane). In our case the brane divides spacetime $M$ into two parts: $M_{1}$ and $M_{2}$. This makes convenient the usage of the symbol $M_{p}$ to indicate integral domains of (II.4) (this is why $p=1$ or $p=2$ ). It should be noticed that $\frac{n^{d}}{}$ changes its direction into the opposite, if the calculations are done on the other side of the brane (that is if $p$ changes its value from 1 to 2 ), while the vector $n^{a}$ remains the same.

Let us now briefly discuss this problem in a more detailed way. At first, assume that $n^{a}$ is "outward pointing" for $p=1$. Such an assumption implies that $n^{a}$ is an "inward pointing" for $p=2$. If, for $p=1$, the vector $n^{a}$ is spacelike (the case of a timelike brane), then $\underline{n^{a}}$ is an "outward pointing", and so $n^{a}=\underline{n^{a}}, n^{a} \underline{n_{a}}=1$. If, for $p=1$, the vector $n^{a}$ is timelike (the case of a spacelike brane), then $\underline{n^{a}}$ is "inward pointing", and so $n^{a}=-\underline{n^{a}}, n^{a} n_{a}=1$. Similarly, if for $p=2$, the vector $n^{a}$ is spacelike (the case of a timelike brane), then $\underline{n^{a}}$ is "outward pointing", and so $n^{a}=-\underline{n^{a}}$ (because in the case of $p=2, n^{a}$ is "inward pointing"), $n^{a} \underline{n_{a}}=-1$. If, for $p=2$, the vector $n^{a}$ is timelike (the case of a spacelike brane), then $\underline{n^{a}}$ is "inward pointing", and so $n^{a}=\underline{n^{a}}, n^{a} \underline{n_{a}}=-1$. It should also be stressed that, for 
$p=1$, we have $h^{a c} \underline{n}_{d}=\epsilon K_{d}^{a}$, and for $p=2$, we have $h^{a c} \underline{n}_{\text {ic }}=-\epsilon K_{d}^{a}[1]$. Unifying these considerations one can write that

$$
\begin{aligned}
n^{a} & =-(-1)^{p} \epsilon \underline{n^{a}}, \\
n^{a} \underline{n}_{a} & =-(-1)^{p}, \\
h^{a c} \underline{n}_{j} ; c & =-(-1)^{p} \epsilon K_{d}^{a} .
\end{aligned}
$$

The last term of the formula (II.4) contains the first derivatives of the metric variation $\delta g_{a b ; c}$, and in the Gibbons-Hawking approach [2] these derivatives should not necessarily be imposed to vanish. Instead, one adds an appropriate boundary term, which cancels the derivatives. The problem is to find its explicit form. In order to do so, let us first choose the foliation fulfilling the condition that $n^{a} n_{b ; a}=0$. Using this, one can derive the following identity

$$
X_{; c}^{c}=D_{e}\left\{h^{e a} X_{a}\right\}+\epsilon K n_{a} X^{a}+\epsilon \mathcal{L}_{\vec{n}}\left(n_{a} X^{a}\right),
$$

where $D_{e}$ is a covariant derivative on the brane and $\mathcal{L}_{\vec{n}}$ is the Lie derivative in the direction of the vector field $\vec{n}$. If we take $X_{c}$ as

$$
X^{c}=2 H g^{a[b} g^{c] d} \underline{n_{d}} \delta g_{a b}
$$

then we have

$$
n_{a} X^{a}=-(-1)^{p} H h^{a b} \delta g_{a b} .
$$

With the help of (II.7), the formula (II.5) can be expressed as

$$
\begin{aligned}
X_{; c}^{c} & =D_{e}\left\{h^{e a} X_{a}\right\}-(-1)^{p} \epsilon K H h^{a b} \delta g_{a b} \\
& -(-1)^{p} \epsilon\left\{\mathcal{L}_{\vec{n}} H\right\} h^{a b} \delta g_{a b} \\
& -(-1)^{p} \epsilon H\left\{\mathcal{L}_{\vec{n}} h^{a b}\right\} \delta g_{a b} \\
& -(-1)^{p} \epsilon H h^{a b}\left\{\mathcal{L}_{\vec{n}} \delta g_{a b}\right\} .
\end{aligned}
$$

Using the identities

$$
\begin{aligned}
\mathcal{L}_{\vec{n}} h^{a b} & =-2 K^{a b}, \\
h^{a b}\left\{\mathcal{L}_{\vec{n}} g_{a b}\right\} & =2 K \\
h^{a b}\left\{\mathcal{L}_{\vec{n}} \delta g_{a b}\right\} & =h^{a b} \delta\left\{\mathcal{L}_{\vec{n}} g_{a b}\right\}+\epsilon K n^{a} n^{b} \delta g_{a b} \\
& -2 D_{b}\left\{h_{a}^{b} \delta n^{a}\right\}, \\
\delta h^{a b}\left\{\mathcal{L}_{\vec{n}} g_{a b}\right\} & =-2 K^{a b} \delta g_{a b}, \\
\delta \sqrt{-h} & =\frac{1}{2} \sqrt{-h} h^{a b} \delta g_{a b},
\end{aligned}
$$

and inserting them into (II.8), after integrating out pure divergencies, we obtain:

$$
\begin{aligned}
X_{; c}^{c} & =-(-1)^{p} \sqrt{-h}\left\{\epsilon h^{a b} H_{; c} n^{c}\right. \\
& \left.+H K n^{a} n^{b}+2 \epsilon n^{b} h^{a e} D_{e} H\right\} \delta g_{a b} \\
& +2(-1)^{p} \sqrt{-h} \epsilon K \delta H \\
& -\delta\left\{2(-1)^{p} \sqrt{-h} \epsilon H K\right\},
\end{aligned}
$$

The last term in (II.10) is exactly what we need to cancel the derivatives of the metric variation $\delta g_{a b ; c}$. In fact, this is exactly the Gibbons-Hawking boundary term which should be appended to the action (II.1) [3]. It reads as

$$
S_{G H, p}=-2(-1)^{p} \epsilon \int_{\partial M_{p}} \sqrt{-h} H K d^{D-1} x .
$$

Bearing in mind that

$$
\begin{aligned}
2 H \underline{n_{d}} ; c g^{a[b} g^{c] d} & =-(-1)^{p} \epsilon H\left(K g^{a b}-K^{a b}\right), \\
4 H_{;(c} \underline{n_{d)}} g^{a[b} g^{c] d} & =-2(-1)^{p} \epsilon\left[g^{a b} H_{; c} n^{c}-H^{;(a} n^{b)}\right],
\end{aligned}
$$

the variation of the full action (supplemented with the boundary term $S_{G H, p}$ )

$$
S_{s, p}=\bar{S}_{p}+S_{G H, p}
$$

gives

$$
\begin{aligned}
\delta S_{s, p} & = \\
& =-\int_{\partial M_{p}} d^{D-1} x \sqrt{-h}(-1)^{p}\left\{\epsilon \left[\left(g^{a b}+\epsilon n^{a} n^{b}\right) \mathcal{L}_{\vec{n}} H\right.\right. \\
& \left.+2 n^{b} h^{e a} H_{, e}+H K h^{a b}-H K^{a b}-2 n^{(a} H^{, b)}\right] \\
& \left.+\frac{\chi}{2} S^{a b}\right\} \delta g_{a b} \\
& -2(-1)^{p} \epsilon \int_{\partial M_{p}} d^{D-1} x \sqrt{-h} K \delta H,
\end{aligned}
$$

where the bulk parts have been omitted. Full variation over the bulk space, separated by a brane, requires the variation of both of these parts separately (i.e. first for $p=1$, and then for $p=2$ ). This means that the full action is

$$
\bar{S}_{p}=S_{s, p=1}+S_{s, p=2}+S_{\text {brane }},
$$

where

$$
\delta S_{\text {brane }}=\int_{\partial M_{p}} d^{D-1} x \sqrt{-h} \frac{\chi}{2} S^{a b} \delta g_{a b} .
$$

After varying the total action (II.14), we obtain the following junction conditions

$$
\begin{aligned}
& -\left(g^{a b}+\epsilon n^{a} n^{b}\right)\left[H_{; c} n^{c}\right]-2 n^{(a} h^{e b)}\left[H_{, e}\right] \\
& -[H K] h^{a b}+\left[H K^{a b}\right]+2 n^{(a}\left[H^{, b)}\right]=\epsilon \frac{\chi}{2} S^{a b},
\end{aligned}
$$

where for any quantity $A$ we have defined

$$
[A] \equiv A^{+}-A^{-} \text {. }
$$

After some manipulations, the Eq. (II.16) can be decomposed into the following set of conditions:

$$
\begin{aligned}
{[K] } & =0 \\
S^{a b} n_{a} n_{b} & =0 \\
S^{a b} h_{a c} n_{b} & =0 \\
-(D-1)\left[H_{; c} n^{c}\right]-D[H] K & =\epsilon \frac{\chi}{2} S^{a b} h_{a b}, \\
-h_{a b}\left[H_{; c} n^{c}\right]-[H] K h_{a b} & +\left[H K_{a b}\right] \\
& =\epsilon \frac{\chi}{2} S^{c d} h_{c a} h_{d b} .
\end{aligned}
$$


These are the most general junction conditions for $f(R)$ gravity on the brane. A physical example of a model which allows the discontinuity of the scalaron $[H] \neq 0$ on the brane is the model which possesses two different cosmological constants $\left(\Lambda_{1}\right.$ and $\left.\Lambda_{2}\right)$ on each side of the brane. In such a case, the Ricci scalar is discontinuous and so the scalaron $H=f^{\prime}(R)$ is discontinuous, too.

After additional assumption of the continuity of the scalaron at the brane position, i.e., after taking

$$
[H]=0
$$

one gets less general junction conditions which were obtained in Refs. [33, 34]:

$$
\begin{aligned}
{[K] } & =0 \\
{\left[H_{; c} n^{c}\right] } & =-\epsilon \frac{\chi}{2(D-1)} S^{a b} h_{a b} \\
H\left[K_{a b}\right] & =\epsilon \frac{\chi}{2}\left\{S^{c d} h_{c a} h_{d b}-\frac{h_{a b} h_{c d}}{(D-1)} S^{c d}\right\} .
\end{aligned}
$$

\section{GIBBONS-HAWKING BOUNDARY TERM AND JUNCTION CONDITIONS FOR F(X,Y,Z) GRAVITY.}

In Refs. [29, 30] a very general fourth-order gravity theory

$$
S=\frac{1}{\chi} \int_{M} d^{D} x \sqrt{-g} f\left(R, R_{a b} R^{a b}, R_{a b c d} R^{a b c d}\right)
$$

has been studied. It has been shown that such a theory allows basic cosmological solutions such as Einstein, deSitter and Gödel universes. Motivated by this result, we now develop similar theory, but within the framework of brane universes. As it has already been said, the main problem to start cosmological solutions is to formulate the appropriate Israel junction conditions for such a theory. This is what we are going to do now.

In order to achieve the task, we apply the GibbonsHawking boundary term method to D-dimensional brane universes, given by the action [26]

$$
\begin{aligned}
S & =\frac{1}{\chi} \int_{M} d^{D} x \sqrt{-g} f\left(R, R_{a b} R^{a b}, R_{a b c d} R^{a b c d}\right) \\
& +S_{\text {brane }}+S_{\text {bulk,p }}
\end{aligned}
$$

where $R, R_{a b} R^{a b}, \quad R_{a b c d} R^{a b c d}$ are curvature invariants, and $\chi$ is a constant. This generalized fourthorder theory includes the first Euler density theory: $f\left(R, R_{a b} R^{a b}, R_{a b c d} R^{a b c d}\right)=\chi \kappa_{1} R$, as well as the second Euler density theory (Gauss-Bonnet term): $f\left(R, R_{a b} R^{a b}, R_{a b c d} R^{a b c d}\right)=\chi \kappa_{2}\left(R_{a b c d} R^{a b c d}-4 R_{a b} R^{a b}+\right.$ $\left.R^{2}\right)\left(\kappa_{1}, \kappa_{2}=\right.$ const.) [6], as special cases. In order to discuss the boundary terms for the action (III.2), we notice that it is still a special case of a more general theory with the action in the form [36]

$$
S_{G}=\chi^{-1} \int_{M} d^{D} x \sqrt{-g} f\left(g_{a b}, R_{a b c d}\right) .
$$

Following the pattern of the $f(R)$ theory given in Section III. we notice that (III.2) is equivalent to the following second-order theory

$$
\begin{aligned}
S_{I} & =\chi^{-1} \int_{M} d^{D} x \sqrt{-g}\left\{H^{g h i j}\left(R_{g h i j}-\phi_{g h i j}\right)\right. \\
& \left.+f\left(g_{a b}, \phi_{c d e f}\right)\right\}
\end{aligned}
$$

where

$$
H^{g h i j} \equiv \frac{\partial f\left(g_{a b}, \phi_{a b c d}\right)}{\partial \phi_{g h i j}}
$$

and

$$
\operatorname{det}\left[\frac{\partial^{2} f\left(g_{a b}, \phi_{a b c d}\right)}{\partial \phi_{g h i j} \partial \phi_{k l m n}}\right] \neq 0 .
$$

The condition (III.6) allows to get an equation of motion of the $\phi_{k l m n}$ field as $\phi_{k l m n}=R_{g h i j}$ in a similar manner as for the $Q$ field in the previous Section [I] In fact, here the tensor $H^{g h i j}$ generalizes the scalaron $H=f^{\prime}(Q)$ of (II.2) onto a tensorial quantity. Because of that, we will call it a tensoron. Varying the equivalent action (III.4), we get the boundary terms in the form

$$
\begin{gathered}
\chi^{-1}\left\{\int_{\partial M_{p}} d^{D-1} x \sqrt{-h} A^{(a b) c d} n_{d} \delta g_{a b ; c}\right. \\
\left.-\int_{\partial M_{p}} d^{D-1} x \sqrt{-h} A_{; d}^{(a b) c d} n_{c} \delta g_{a b}\right\},
\end{gathered}
$$

where

$$
\begin{aligned}
A^{a b c d}=\frac{1}{2}\left\{H^{a c d b}\right. & +H^{a b d c}-H^{c b d a}-H^{a c b d} \\
& \left.-H^{a b c d}+H^{c b a d}\right\} .
\end{aligned}
$$

In a special case of the $f\left(R, R_{a b} R^{a b}, R_{a b c d} R^{a b c d}\right)$ theory, the tensor $A^{a b c d}$ reads as

$$
\begin{aligned}
A^{a b c d} & =f_{X}\left(g^{a d} g^{c b}-g^{c d} g^{b a}\right) \\
& +f_{Y}\left(2 R^{a d} g^{b c}-R^{c d} g^{b a}-R^{b a} g^{c d}\right) \\
& +4 f_{Z} R^{a c b d}
\end{aligned}
$$

Finally, an appropriate Gibbons-Hawking boundary term for the action (III.4 is

$$
\begin{aligned}
S_{G H, p} & = \\
& -(-1)^{p} \int_{\partial M_{p}} d^{D-1} x \sqrt{-h} A^{(a b) c d} n_{c} n_{d} \mathcal{L}_{\vec{n}} g_{a b} .
\end{aligned}
$$

Introducing $X^{c}=A^{(a b) c d} n_{d} \delta g_{a b}$ into (II.5), and applying the relations

$$
\mathcal{L}_{\vec{n}} \delta g_{a b}=\delta\left\{\mathcal{L}_{\vec{n}} g_{a b}\right\}-g_{e b}\left\{\delta n^{e}\right\}_{; a}-g_{e a}\left\{\delta n^{e}\right\}_{; b}
$$

$$
A^{(a b) c d} n_{c} n_{d} n_{b}=0
$$


where the second equation (III.12) is fulfilled for $A^{(a b) c d}$ given by (III.9), one obtains the following junction conditions for the $f\left(R, R_{a b} R^{a b}, R_{a b c d} R^{a b c d}\right)$ brane gravity:

$$
\begin{aligned}
& {\left[K A^{(a b) c d}\right] n_{c} n_{d}+\left[\mathcal{L}_{\vec{n}} A^{(a b) c d}\right] n_{c} n_{d} } \\
- & \epsilon\left[A^{(a b) c d} K_{c d}\right]-g^{a b}\left[A^{(e f) c d} K_{e f}\right] n_{c} n_{d} \\
+ & 2 \epsilon\left[D_{s} A^{(e f) c d} n_{c} n_{d}\right] h_{e}^{s} h_{f}^{(a} n^{b)}-2 \epsilon\left[A^{(a b) c d} ;(c] n_{d)}=\frac{\chi}{2} S^{a b},\right. \\
& n_{b} n_{c}\left[\mathcal{L}_{\vec{n}} g_{a d}\right]-n_{a} n_{c}\left[\mathcal{L}_{\vec{n}} g_{d b}\right]-n_{b} n_{d}\left[\mathcal{L}_{\vec{n}} g_{a c}\right] \\
+ & n_{a} n_{d}\left[\mathcal{L}_{\vec{n}} g_{c b}\right]=0 .
\end{aligned}
$$

These junction conditions coincide with those obtained by the application of a different method in our previous Ref. [26] (Eqs. (5.4)-(5.5)), provided that

$$
\left[A^{(a b) c d}\right]=0,
$$

i.e., after the assumption that the tensoron is continuous at the brane position. This is an analogous condition to the condition for the continuity of the scalaron on the brane (II.23) in the $f(R)$ theory of brane gravity.

\section{SUMMARY}

In this paper we derived the most general junction conditions for both $f(R)$ and $f\left(R, R_{a b} R^{a b}, R_{a b c d} R^{a b c d}\right)$ braneworld gravities by the application of the GibbonsHawking boundary term method. We generalized previously obtained junction conditions for $f(R)$ gravity for the case in which we did not assume the continuity of the scalaron field $H=f^{\prime}(Q)$ on the brane. Such a case appears, for example, if one takes two different values of the cosmological constants in the bulk on each side of the brane. After assuming the continuity of the scalaron, these most general junction conditions reduce to the ones obtained in earlier references [33, 34]. Next, we derived the most general junction conditions for the $f\left(R, R_{a b} R^{a b}, R_{a b c d} R^{a b c d}\right)$ braneworld gravity. Here, we also did not make any assumption about the continuity of the tensoron field $A^{(a b) c d}$ at the brane position. Again, we have shown that these junction conditions reduce to those obtained by different methods in our earlier paper [26], provided one assumes the continuity of the tensoron field at the brane. We should stress that even if the scalaron and tensoron fields are discontinuous on the brane, and therefore their normal derivatives produce a delta function singularity on the brane, the quantity which determines a jump of the normal derivative of the scalaron or the tensoron on the brane is well-defined (e.g. in the case of delta function having a singularity at some point $x_{0}$, a jump of delta function $x_{0}$ is equal to zero - this comes directly form the definition of the limit of the function). This obviously means that neither the scalaron nor the tensoron have to be continuous across the brane, so that the junction conditions (II.18)-(II.22) and (III.13)-(III.14) are physically realistic.

We think that the method of the Gibbons-Hawking boundary term is more elegant than other methods of deriving junction conditions, provided one is able to suggest a correct boundary term. Up to our knowledge, so far, only the gravity theory of the linear combination of the curvature invariants $f\left(R, R_{a b}, R_{a b c d}\right)=$ $a R^{2}+b R_{a b} R^{a b}+c R_{a b c d} R^{a b c d}(a, b, c=$ const.), was studied in the Gibbons-Hawking boundary term approach [35].

We consider our result as a basic step in order to write down cosmological equations for $f\left(R, R_{a b}, R_{a b c d}\right)$ brane gravity and to check, if basic csomological models are allowed in such a framework. Besides, the discontinuity of the scalaron and tensoron on the brane can be used to describe some other physical cases of the surface layers, such as boundary surfaces separating stars from the surrounding vacuum (singular hypersurfaces of higher order [17]).

We also hope that the analysis of the higher-order brane cosmologies will give some characteristic pattern in statefinder (jerk, kerk/snap, lerk/crackle, merk/pop) diagnostic of cosmology [37], in a similar manner as it was given in Ref. 38] for $f(R)$ non-brane gravity models. This, however, will be the matter of a separate paper [39].

\section{ACKNOWLEDGMENTS}

We thank Andrei Barvinsky, Salvatore Capozziello, Tomasz Denkiewicz, Krzysztof Meissner and David Wands for discussions. We acknowledge partial support of the Polish Ministry of Science and Higher Education grant No N N202 191234 (years 2008-10).
[1] Wald R., General Relativity (University of Chicago Press, 1984).

[2] G.W. Gibbons and S.W. Hawking, Phys. Rev. D 15, 2752 (1977).

[3] S.W. Hawking and J.C. Lutrell, Nucl. Phys. B247, 250 (1984).

[4] M. Madsen and J.D. Barrow, Nucl. Phys. B323, 242 (1989).
[5] A.D. Barvinsky and S.N. Solodukhin, Nucl. Phys. B 479, 305 (1996).

[6] D. Lovelock, J. Math. Phys. 12, 498 (1971).

[7] C.C. Briggs, gr-qc/9808050

[8] T.S. Bunch, Journ. Phys. A 14, L139 (1981).

[9] F. Müller-Hoissen, Phys. Lett. B 163, 106 (1985); R.C. Myers, Phys. Rev. D 36, 392 (1987).

[10] S.C. Davis, Phys. Rev. D 67, 024030 (2003). 
[11] E. Gravanis and S. Willinson, Journ. Math. Phys. 47, 2503 (2006); Phys. Rev. D75, 084025 (2007).

[12] O. Mišković and R. Olea, JHEP 0710, 028 (2007).

[13] P. Hořava and E. Witten, Nucl. Phys. B460 (1996), 506; ibid B475, 94.

[14] L. Randall and R. Sundrum, Phys. Rev. Lett., 83, 3370 (1999); L. Randall and R. Sundrum, ibidem, 83, 4690 (1999).

[15] M. Visser, Phys. Lett. B159, 22 (1985); N. ArkaniHamed, S. Dimopoulos, and G. Dvali, Phys. Lett. B516, 70 (1998); I. Antoniadis, N. Arkani-Hamed, S. Dimopoulos, G. Dvali, Phys. Lett. B436, 257 (1998); N. ArkaniHamed, S. Dimopoulos, and G. Dvali, Phys. Rev. D59, 086004 (1999).

[16] M. Visser, Lorentzian Wormholes (Springer-Verlag, 1996).

[17] W. Israel, Nuovo Cimento B 44, 1 (1966).

[18] P. Binétruy, C. Deffayet and D. Langlois, Nucl. Phys. B565, 269 (2000); P. Binétruy, C. Deffayet and D. Langlois, Phys. Lett. B477, 285 (2000); M. Sasaki, T. Shiromizu and K. Maeda, Phys. Rev. D62, 024008 (2000); T. Shiromizu, K.I. Maeda, and M. Sasaki, Phys. Rev. D62, 024012 (2000); S. Mukhoyama, T. Shiromizu and K. Maeda, Phys. Rev. D62, 024028 (2000).

[19] M. Arik and D. Çiftci, Gen. rel. Grav. 37, 2211 (2005); M.C.B. Abdalla, M.E.X. Guimarães, and J.M. Hoff de Silva, hep-th/0711.1254.

[20] N. Deruelle and T. Doležel, Phys. Rev. D62, 103502 (2000).

[21] C. Charmousis, J.F. Dufaux, Class. Quantum Grav. 19, 4671 (2002).

[22] J.F. Dufaux, J.E. Lidsey, R. Maartens, and M. Sami, Phys. Rev. D 70, 083525 (2004).

[23] J.E. Lidsey, Ann. Phys. (Leipzig) 15, 277 (2006).

[24] H. Maeda, V. Sahni, Yu. Shtanov, Phys. Rev. D 76, 104028 (2007).

[25] K.A. Meissner and M. Olechowski, Phys. Rev. Lett. 86, 3708 (2001).

[26] A. Balcerzak and M.P. Dąbrowski, Phys. Rev. D77, 023524 (2008).

[27] A.A. Starobinsky, Phys. Lett. B 91, 99 (1980); G. Magnano and L.M. Sokołowski, Phys. Rev. D 50, 5039 (1994); T.P. Sotiriou, Class. Quantum Grav. 23, 5117 (2006); T. Chiba, T. Kobayashi, M. Yamaguchi, and J. Yokoyama, Phys. Rev. D 75, 043516 (2007); G.J. Olmo, Phys. Rev.
Lett. 98, 061101 (2007); G.J. Olmo, Phys. Rev. D 75, 023511 (2007); S. Capozziello, V.F. Cardone, and A. Troisi, Phys. Rev. D 71, 043503 (2005); S. Capozziello, S. Nojiri, S.D. Odintsov, and A. Troisi, Phys. Lett. B 639, 135 (2006); S. Capozziello and R. Garatini, Class. Quantum Grav. 24, 1627 (2007); L. Amendola, D. Polarski, and S. Tsujikawa, Phys. Rev. Lett. 98, 131302 (2007); S. Nojiri and S.D. Odintsov, arXiv: 0804.3519; 0807.0685; T. Faulkner, M. Tegmark, E.F. Bunn, and Y. Mao, Phys. Rev. D 76, 063505 (2007); N. LanahanTremblay and V. Faraoni, hep-th/0709.4414; Q. Exirifard, gr-qc/0708.0662; S. Capozziello, V.F. Cardone, and A. Troisi, astro-ph/0604435

[28] J. Sotiriou and V. Faraoni, arXiv:0805.1726.

[29] T. Clifton and J.D. Barrow, Phys. Rev. D 72, 123003 (2005); T. Clifton and J.D. Barrow, Class. Quantum Grav. 23, 2951 (2006).

[30] J.D. Barrow and S. Hervik, Phys. Rev. D 73, 023007 (2006); ibidem, 74, 124017 (2006).

[31] A. Frolov, astro-ph/0803.2500.

[32] H.H. v. Borzeszkowski and V.P. Frolov, Ann. Phys. (Leipzig) 7, 285 (1980).

[33] M. Parry, S. Pichler, and D. Deeg, JCAP 0504, 014 (2005).

[34] N. Deruelle, M. Sasaki, and Y. Sendouda, grqc/0711.1150.

[35] S. Nojiri and S.D. Odintsov, JHEP 0007, 049 (2000); S. Nojiri, S.D. Odintsov and S. Ogushi, Phys. Rev. D 65, 023521 (2001).

[36] A. Jakubiec and J. Kijowski, Phys. Rev. D 37, 1406 (1988).

[37] V. Sahni, T.D. Saini, A.A. Starobinsky, and U. Alam, JETP Lett. 77, 201 (2003); M. Visser, Class. Quantum Grav. 21, 2603 (2004); R.R. Caldwell and M. Kamionkowski, JCAP 0409, 009 (2004); M.P. Dąbrowski, Phys. Lett. B625, 184 (2005); M.P. Dąbrowski and T. Stachowiak, Annals of Physics (New York) 321, 771 (2006); M. Dunajski and G.W. Gibbons, arXiv: 0807.0207.

[38] N.J. Poplawski, Phys. Lett. B640, 135 (2006); Class. Quantum. Grav. 24, 3013 (2007); S. Capozziello, V.F. Cardone, and V. Salzano, astro-ph/0802.1583.

[39] A. Balcerzak and M.P. Dąbrowski - in preparation. 